\title{
From zero to six double bonds: phospholipid unsaturation and organelle function
}

\author{
Bruno Antonny ${ }^{1}$, Stefano Vanni ${ }^{1}$, Hideo Shindou ${ }^{2,3}$, and Thierry Ferreira ${ }^{4}$ \\ ${ }^{1}$ Institut de Pharmacologie Moléculaire et Cellulaire, Université de Nice Sophia Antipolis and Centre National de la Recherche \\ Scientifique (CNRS), 660 route des Lucioles, 06560 Valbonne, France \\ ${ }^{2}$ Department of Lipid Signaling, Research Institute, National Center for Global Health and Medicine, 1-21-1 Toyama, Shinjuku-ku, \\ Tokyo 162-8655, Japan \\ ${ }^{3}$ CREST, Japan Science and Technology Agency, Kawaguchi, Saitama 332-0012, Japan \\ ${ }^{4}$ Laboratoire Signalisation et Transports loniques Membranaires (STIM), CNRS Equipe de Recherche Labellisée (ERL) 7368, \\ Université de Poitiers, 1 rue Georges Bonnet, 86073 Poitiers CEDEX 9, France
}

Cellular phospholipids (PLs) differ by the nature of their polar heads as well as by the length and unsaturation level of their fatty acyl chains. We discuss how the ratio between saturated, monounsaturated, and polyunsaturated PLs impacts on the functions of such organelles as the endoplasmic reticulum, synaptic vesicles, and photoreceptor discs. Recent experiments and simulations suggest that polyunsaturated PLs respond differently to mechanical stress, including membrane bending, than monounsaturated PLs owing to their unique conformational plasticity. These findings suggest a rationale for PL acyl chain remodeling by acyltransferases and a molecular explanation for the importance of a balanced fatty acid diet.

Fatty acyl chain diversity: the dark face of cellular membranes

The lipid composition of cellular membranes varies significantly among organisms, tissues, and organelles, and our knowledge of this diversity has greatly increased thanks to progress in organelle fractionation, lipid analysis, notably mass spectroscopy, and the identification and characterization of most enzymes responsible for lipid synthesis [15]. However, our understanding of the roles of the various lipids that coexist in biological membranes has not improved as fast. Hundreds of different lipid species can be found in the membrane of a single organelle, but we can attribute a clear function to only a few of them.

With the exception of sterols, the lipids of cellular membranes are made of three building blocks: a polar head, a central group (glycerol or sphingosin), and long hydrocarbon chains (the fatty acids). This scheme allows numerous combinations and is at the root of lipid diversity. However, biochemists and cell biologists generally do care more about the polar head than about the acyl chains. This

Corresponding author: Antonny, B. (antonny@ipmc.cnrs.fr).

Keywords: polyunsaturated phospholipid; membrane curvature; lipid-packing defect; mechanotransduction; lipid remodeling. bias is reflected in the language: we name lipids by their polar head (e.g., phosphatidylcholine, PC; phosphatidylserine, PS; phosphatidylethanolamine, PE), but we rarely detail their acyl chain content. It also acknowledges the fact that lipid polar heads are associated with defined functions owing to their specific interactions with proteins [6].

The role of the various fatty acyl chains of PLs is less well understood. Fatty acids are divided into three classes according to the number of double carbon bonds along the carbon chain (Figure 1A) [7]. Saturated fatty acids (SFA) contain only saturated carbon bonds $(\mathrm{C}-\mathrm{C})$; monounsaturated fatty acids (MUFA) contain a single cis double bond $(\mathrm{C}=\mathrm{C})$; polyunsaturated fatty acids (PUFA) contain 2-6 cis double bonds and are themselves divided into two subclasses, $\omega 3$ and $\omega 6$, depending on the position of the double bonds. Palmitate, oleate, arachidonate, and docosahexanoic acid (DHA) are the most abundant fatty acids of each class. Their abbreviation (C16:0, C18:1-n9, C20:4-n6, and C22:6-n3, respectively) indicates the number of carbons, the number of double bonds, and the position of the first double bond with respect to the terminal methyl.

On average, the first acyl chain of cellular PLs is saturated whereas the second acyl chain is mono or polyunsaturated. However, there are large variations around this canonical scheme. Why is the endoplasmic reticulum (ER) richer in monounsaturated PLs than the plasma membrane (PM) $[8,9]$ ? Why are polyunsaturated PLs so abundant in some specialized organelles (e.g., synaptic vesicles) but absent in some organisms (e.g., Saccharomyces cerevisiae) [9-12]? These questions, which echo back to the discovery in the early 1930s of the essential role of some PUFAs in mammalian development [13], are still topical: we frequently hear that olive oil is better than butter and that $\omega 3$ are better than $\omega 6$ lipids for our health, but the molecular basis for these differences remains elusive.

Fatty acids could have effects on their own or as precursors of signaling molecules [14]. In addition, because fatty acids become in large part esterified in PLs, they should impact on the functioning of cellular membranes [10]. We summarize here the origin of the acyl chain 


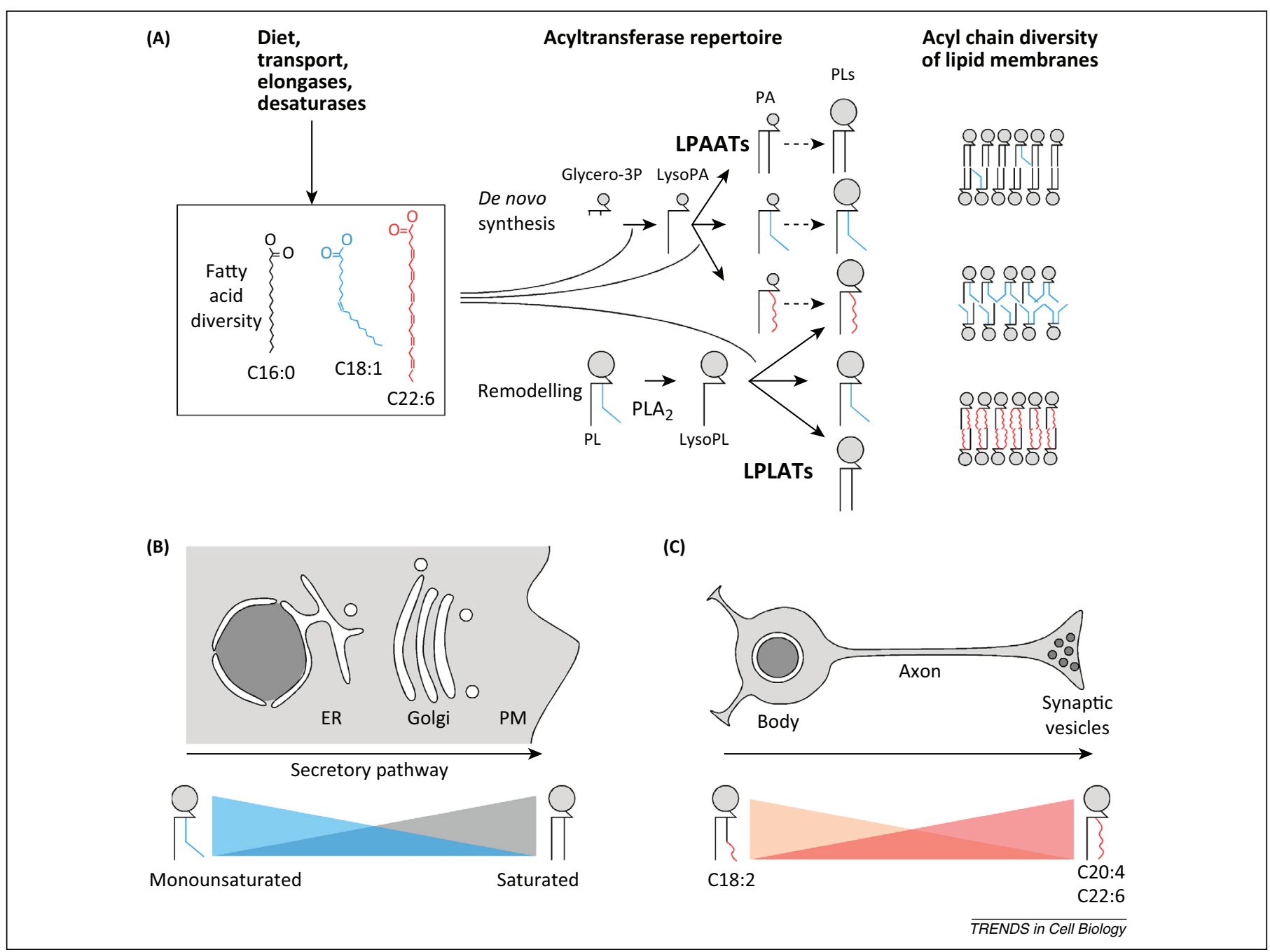

Figure 1. Acyl chain diversity of membrane lipids. (A) Summary of the various lipid metabolic pathways that lead to the acyl chain diversity of phospholipids (PLs) in biological membranes. The diversity of fatty acids arises from dietary sources, as well as from endogenous enzymatic activities that allow the acyl chain to be elongated or desaturated. In many organisms, however, these activities are limited, hence the importance of a diverse diet. Fatty-acyl-CoA intermediates are then used as substrates by various lysophospholipid acyltransferases to synthesize or remodel PLs $[5,15]$. These enzymes form two large families and include members displaying different specificity for their lysophospholipid and acyl-CoA substrates (Box 1). (B,C) The acyl chain profile of PLs varies along the organelles of the secretory pathway as well as in some differentiated structures such as axons $[9,12]$.

diversity in PLs and present a few examples of mutual adaptation between organelle function and PL acyl chain composition. Last, we discuss the importance of biophysical approaches coupled to molecular dynamics simulations to reveal how deceptively simple changes in the hydrocarbon structure of PL fatty acids can impart biological membranes with different physicochemical properties.

\section{Biochemical pathways promoting PL acyl chain diversity}

The acyl chain diversity of PLs results from several processes, from diet sources to complex reactions where fatty acids are elongated, desaturated, transported, and eventually esterified into PLs (Figure 1A) [15,16].

Almost all organisms contain a $\Delta 9$ desaturase, which introduces a double bond in the middle of the acyl chain, thus producing monounsaturated fatty acids from saturated ones (e.g., C18:0 > C18:1-n9). By contrast, the ability to add additional double bonds is not universal. Some plants introduce double bonds between the terminal methyl and the central carbon, whereas mammals introduce double bonds between the central carbon and the terminal carboxyl [7]. Our diet reflects this division of labor: we eat the essential linolenic acid C18:3-n3 from plant oils to synthesize C22:6-n3, which then accumulates in our brain membranes. A transporter allowing C22:6-n 3 to cross the brainblood barrier has been recently identified and its deletion strongly impairs brain development [17].

Fatty acids are eventually activated in the form of acyl-CoA intermediates and esterified into PLs through two alternative pathways: the de novo pathway (or Kennedy pathway) corresponds to the complete synthesis of a PL from elementary building blocks, whereas the remodeling pathway (or Lands cycle) corresponds to the mere substitution of one acyl chain in a preformed PL (Figure 1A) [18]. The acyltransferases involved in these pathways have been identified and their substrate specificity determines the acyl chain diversity of PLs (Box 1) [15]. Striking examples are LPCAT1, which is enriched in the lung and incorporates saturated fatty acids into 
The fatty acid composition at both $s n-1$ and $s n-2$ positions of PLs differs in various cell types and tissues. Asymmetry and diversity of membrane PLs are generated through the de novo pathway and the remodeling pathway [18]. PLs are formed from glycerol-3-phosphate (G3P) by the Kennedy pathway. Next, using acyl-CoAs as donors, the sn-2 acyl moiety of PLs is remodeled in the Lands' cycle, which is conducted by the concerted actions of phospholipase A2 (PLA2) and lysophospholipid acyltransferase (LPLAT) enzymes. The Kennedy pathway and the Lands' cycle were proposed in the 1950s. However the corresponding LPLATs were discovered only recently [15]. LPLATs are divided into two families: the 1-acylglycerol-3-phosphate $O$ acyltransferase (AGPAT) family and the membrane-bound $\mathrm{O}$-acyltransferase (MBOAT) family.

In the AGPAT family, GPAT1-4 and LPAAT1-4 produce lysophosphatidic acid (LPA) and phosphatidic acid (PA) in the Kennedy pathway, respectively, whereas the other members are Lands' cycle enzymes. LPCAT1 is mainly expressed in the lung and generates PAF and dipalmitoyl-PC, which is a main component of pulmonary surfactant essential for respiration [5]. LPCAT2 functions in inflammatory cells to synthesize platelet-activating factor (PAF) and PC. LPCAT2 is phosphorylated and is activated by extracellular stimuli

PC to promote the formation of a protective surfactant [5], and tafazzin, which incorporates polyunsaturated fatty acids into cardiolipin (CL) in a curvature-dependent manner [19].

\section{Examples of PL fatty acyl chain gradients}

(1)

In mammalian cells, there is a gradual enrichment of saturated PL species at the expense of monounsaturated species along the organelles of the secretory pathway (ER $>$ Golgi > plasma membrane) [8]. This subcellular gradient, which parallels main membrane traffic routes, also exists in yeast and results from a change in the esterified acyl chains of PE and PS [9] (Figure 1B).

Recent advances in lipid imaging by mass spectrometry reveal another striking acyl chain gradient (Figure 1C). In neuronal cell cultures, where axons radiate around a cluster of cell bodies, a gradient of polyunsaturated PC has been imaged at $\mu \mathrm{m}$ resolution [12]. PC molecules containing C20:4 or C22:6 PUFAs accumulate at the axon tip but are rare at the cell body, whereas less-unsaturated species, notably C18:2-PC, show an opposite distribution. Spermatozoa provide another example of the uneven distribution of polyunsaturated PLs at the subcellular level [20].

Cells also change their PL acyl chain profile over time. Epithelium differentiation is accompanied by the appearance of more saturated lipid species, probably to make the apical PM a better protective barrier [1]. The amount of C20:4-containing PC oscillates during the cell cycle [21]. During cytokinesis, lipid species with well-defined acyl chain composition appear in the mid body [22]. Last, the ratio between monounsaturated PLs and polyunsaturated PLs increases in several cancer cell lines and tissues $[23,24]$.

The metabolic pathways at the origin of the spatiotemporal control of PL acyl chain unsaturation remain to be investigated, notably the involvement of specific acyltransferases [5]. However, the fact that this control exists, whereas the multiple membrane traffic pathways should lead to PL homogenization [4], suggests that the balance between saturated, monounsaturated, and polyunsaturated PL species has decisive functions. such as ATP, PAF, and lipopolysaccharide [103]. LPEAT2 is expressed in the brain and produces PE. LPGAT1 and LCLAT1 generate phosphatidylglycerol (PG) and CL, respectively. In the MBOAT family, LPCAT3, LPCAT4, LPEAT1, and LPIAT1 have been identified. LPIAT1 synthesizes phosphatidylinositides (PI) containing arachidonic acid, the main forms of PI [94]. LPCAT3 (PC, PE, and PS production), LPCAT4 (PC and PE), and LPEAT1 (PE and PS) possess several enzymatic activities and show different expression patterns, suggesting specific roles in different tissues [15].

$\mathrm{PC}$ is the major PL and displays a highly-diverse fatty acid profile depending on the tissue [5]. Fatty acid enrichment of PC can be regulated by two reactions: the LPAAT step in the Kennedy pathway and the LPCAT step in the Lands' cycle. The molecular mechanism of PC fatty acid determination was examined using LPAAT1, 2, and 3, as well as LPCAT1, 2, 3, and 4. From enzymatic assay and PC composition of several tissues, the LPCAT step seems to control the formation of PC species containing $16: 0$ and $18: 1$ at $s n-2$, whereas the LPAAT step seems to control the incorporation of $18: 2$ and 22:6 in PC at the $s n-2$ position. Further investigations will be necessary to understand the diversity of other PLs, such as PS, PE, PI, PG, and CL. Regulation of fatty acid diversity at the $s n-1$ position should be also clarified.

\section{Influence of PL acyl chains on protein synthesis and folding at the ER}

The ER is the organelle for the biosynthesis and folding of transmembrane and luminal proteins. To maintain the correct balance between ER client protein load and folding capacity, cells have developed a pathway known as the unfolded protein response (UPR) [25,26]. The UPR is controlled by integral protein sensors, such as inositolrequiring enzyme 1 (IRE1) and protein kinase-like ER kinase (PERK) [25,26], which are maintained in an inactive monomeric form by the interaction of their luminal domain with the chaperone BiP. Accumulation of unfolded proteins correlates with the disruption of this repressive complex, oligomerization, and downstream activation of the UPR cascade $[27,28]$. Induction of the UPR limits protein overload via a general decrease in translation initiation and coordinated upregulation of genes encoding proteins involved in folding (e.g., chaperones) and ER-associated degradation (ERAD). Interestingly, these pathways are activated upon cell exposure to SFAs [29-34].

Because exogenously supplied fatty acids are efficiently incorporated into PLs [35-37], several studies point at saturated PLs as the direct cause of ER-stress as a result of their impact on membrane properties. This includes modification of calcium permeation, perturbation of protein folding, and changes in the oligomeric state of IRE1. For example, palmitate promotes depletion of ER calcium stores $[30,38]$ in a process that could be related to direct effects of saturated PLs on sarcoplasmic-endoplasmic reticulum calcium ATPase (SERCA) pump activity [30]. In vitro studies demonstrate that SERCA-2b activity is inhibited when reconstituted into microsomes containing saturated PLs [39]. Saturated PLs also induce the accumulation of misfolded proteins. Treating SFA-intoxicated cells with pharmacological chaperones that stabilize protein conformation alleviates SFA-induced UPR [37,4042]. Various causes could account for misfolded protein accumulation under SFA treatment. First, the translocon, which catalyzes the translocation of newly translated proteins towards the lumen, is sensitive to membrane 
organization [43]. Second, many ER chaperones are dependent on calcium [44], the homeostasis of which is altered under palmitate accumulation.

Intriguingly, recent studies suggest that lipid disequilibrium could induce UPR by a direct effect on IRE1 and PERK $[45,46]$. Mutants of these proteins lacking the luminal unfolded protein-sensing domain still respond to increased lipid saturation [45]. Moreover, increasing PL saturation in reconstituted liposomes that are devoid of unfolded ER client proteins enhanced the activity of a PERK variant devoid of its luminal domain [45]. Even though the mechanism for saturated PL-induced PERK clustering is a matter of debate [47], these observations suggest that misfolded protein accumulation may not be the only signal for UPR induction under SFA accumulation. Dimerization of IRE1 and PERK upon overload of the ER membrane with saturated-PLs might allow the cell to anticipate future protein misfolding problems induced by these lipids. Conversely, the fact that a component of the ERAD machinery, Ubx2p, has been identified as a key activator of yeast $\Delta 9$ desaturase [48] suggests a feedback loop whereby ER stress induced by misfolded proteins promotes correction of the ER unsaturation level.

\section{PL monounsaturation, membrane curvature, and protein adsorption}

How the ratio between saturated and monounsaturated PLs controls transmembrane helices oligomerization at the ER remains elusive, although several mechanisms have been proposed [49]. By contrast, the monounsaturat$\mathrm{ed} /$ saturated PL ratio has another impact that is more straightforward to rationalize. Introducing monounsaturated PLs at the expense of saturated ones facilitates the membrane adsorption of several cytosolic proteins acting on the ER or ER-derived organelles such as autophagosomes or Golgi [2]. In general, these proteins contain large amphipathic membrane anchors, and their preference for monounsaturated PLs correlates with an acute sensitivity to membrane curvature $[2,50,51]$.

The motifs that allow peripheral proteins to sense the combinatory effects of monounsaturated PLs ratio and membrane curvature have been reviewed elsewhere [50]. We recall here the underlying mechanism, which revolves around the idea of 'voids' or lipid-packing defects (Figure 2A). In this simple geometrical model, lipids are considered as rather stiff objects. When membrane curvature increases, or when monounsaturated PLs are substituted for saturated ones, the lipid geometrical arrangement is perturbed and packing defects gradually appear, which could host hydrophobic anchors from proteins [52,53].

Combining the effect of curvature and lipid monounsaturation in vitro can change protein adsorption by two orders of magnitude, suggesting a potent regulatory mechanism [50-52,54]. Testing the relevance of this mechanism in vivo is challenging because it requires mastering both the geometry and lipid composition of cellular membranes. However, recent data are in agreement with the model. Excess levels of saturated PLs hamper processes as diverse as ER-to-Golgi trafficking or autophagosome formation, and promote the membrane dissociation of some key associated proteins [54-57].

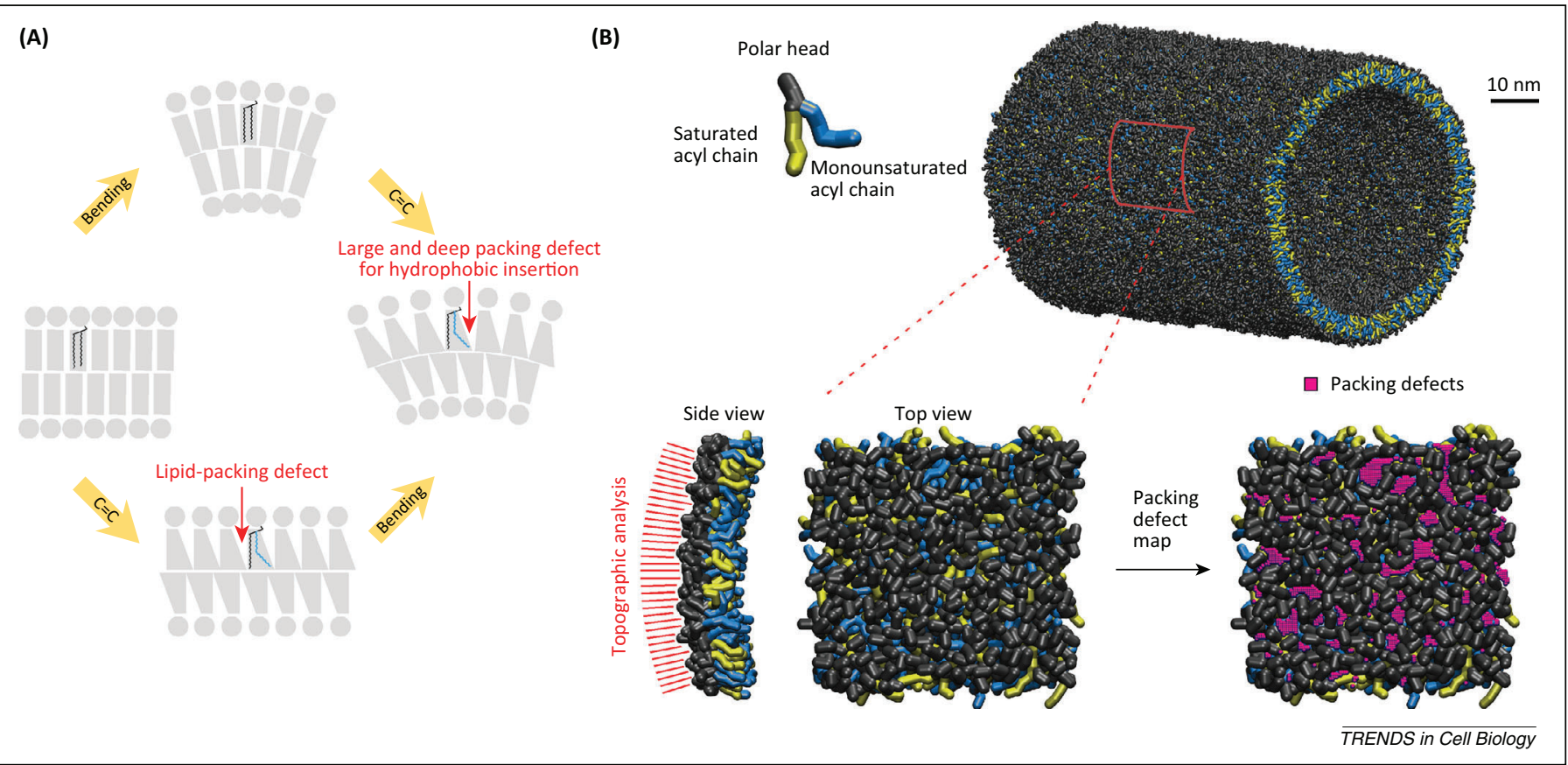

Figure 2. Cooperation between monounsaturated phospholipids (PLs) and curvature for protein insertion. (A) Elementary view of the effects of membrane curvature and lipid monounsaturation on the formation of lipid-packing defects. This naive model considers the gross shape of lipids as well as their relative tilting due to curvature. It accounts, at least qualitatively, for the cumulative effects of membrane curvature and lipid monounsaturation on the adsorption of model amphipathic motifs at the membrane-water interface [50,52,53]. (B) Molecular dynamics simulations give a more realistic view of the effect of lipid composition and membrane shape on the formation of lipid-packing defects [54,58-60]. The example shown here considers a membrane tube made of a single lipid (C16:0-C18:1-PC) that has been 'coarse-grained' to minimize calculations. Scanning the membrane surface allows identifying 'voids' (i.e., lipid-packing defects). The statistical distribution of these voids confirms the cumulative effects of membrane curvature and monounsaturation on lipid-packing defects. 


\section{Molecular dynamics simulations of lipid-packing defects}

Although the scheme of Figure $2 \mathrm{~A}$ is obviously naïve because lipids are not stiff, addressing the molecular organization of lipids in bilayers of different compositions and geometries is experimentally very difficult. To overcome these limitations, and to investigate microscopic properties of lipid assemblies, a powerful methodology is molecular dynamics simulations (Box 2), a computational approach that allows investigating the behavior of lipid membranes with atomic-level resolution. Using this technique it has been possible to characterize lipid-packing defects occurring at the membrane-water interface, thereby providing a topographic description of lipid bilayers [54,58,59] (Figure 2B). Despite being transient (with lifetimes in the picosecond regime), lipid-packing defects are ubiquitous and can be precisely quantified for all types of lipid assemblies. Their size displays a characteristic exponential distribution (large defects are much less frequent than small ones).

Molecular dynamics simulation of varying membrane geometries and compositions show that lipid-packing defects increase with positive curvature, and also with - the introduction of monounsaturated PLs at the expense of saturated ones [54]. Most importantly, curvature and PL monounsaturation have a cumulative effect towards the promotion of lipid-packing defects, whereas curvature alone, in the presence of saturated PLs, is not sufficient to promote membrane adsorption of peripheral proteins, in agreement with experimental data $[54,58-60]$. This example suggests that molecular dynamics simulations can provide an atomic view of an intuitive but elusive model of PL organization, and complements other approaches that consider membranes as continuum material [61]. The simulations show that lipid-packing defects do not necessarily localize at the position of the most conical lipids [59] and need to coalesce to provide enough volume to host large hydrophobic insertions [58]. Nevertheless, saturated and monounsaturated PLs are relatively simple lipids for which a large body of experimental data exists, making their simulations a not too risky task.

\section{PL polyunsaturation in phototransduction}

Phototransduction, one of the best-characterized transduction cascades, occurs in a membrane rich in w3 lipids. In the photoreceptor discs, DHA (22:6-n3) accounts for $50 \%$ of the PL acyl chains [62]. The proteins involved in phototransduction, namely the light receptor rhodopsin, the $\mathrm{G}$ protein transducin, and its effector, a cGMP phosphodiesterase, have been reconstituted into artificial liposomes. This reductionist approach revealed that replacing C16:0-C18:1 by C18:0-C22:6 PLs increases the rates at which rhodopsin switches to the active state and subsequently activates GDP/GTP exchange on transducin $[57,63,64]$. Because the first reaction is a monomolecular process that occurs within the hydrophobic matrix, whereas the second reaction is a bimolecular process that occurs at the surface of photoreceptor discs, these observations suggest that polyunsaturated PLs provide two advantages: they facilitate the movements of the helical segments of rhodopsin and they accelerate in-plane diffusion of peripheral proteins.

\section{Biophysical measurements of the behavior of polyunsaturated PLs in bilayers}

Studies on phototransduction have stimulated detailed biophysical studies on the behavior of polyunsaturated PLs in model membranes [65,66]. Neutron and X-ray diffraction as well as NMR measurements revealed that, in bilayers containing mixed acyl chain PLs (e.g., C16:0C18:1 vs C18:0-C22:6), polyunsaturated acyl chains occupy more space at the water interface than saturated or monounsaturated chains, despite polyunsaturated acyl chains being generally longer (e.g., C22:6 vs C18:1). This counterintuitive finding suggests that polyunsaturated acyl chains are not extended but are curled (Figure 3A). However,

\section{Box 2. Molecular dynamics (MD) simulations}

$\mathrm{MD}$ is a computational methodology that is used to simulate the physical movement of systems composed of several atoms. Originally developed in the context of theoretical physics, this technique is now used in several domains, from physical chemistry to material science, to investigate with high-resolution the behavior of multiatom systems that are difficult or expensive to characterize experimentally.

In a MD simulation, all atoms in the system are treated as classical particles (i.e., quantum effects are neglected), and forces and energies between atoms are described using a so-called molecular mechanics force-field - in other words a simplified potential energy function where the interactions between atoms are subdivided into bonded (bonds, angles, and dihedrals) and non-bonded (electrostatic and Van der Waals) terms. Specifying these interactions requires a large number of parameters (e.g., equilibrium values and force constants for bonds, angles, and dihedrals; electrostatic charges; van der Waals parameters) that are usually obtained from either experiments or high-level quantum mechanical calculations. Once these parameters have been set, the dynamics of the system is studied by numerically integrating the equations of motion, up to the statistical convergence of the properties of interest.

Because of the numerous approximations that are carried out to make the problem tractable, the results from MD need to be validated by experimental data. When the agreement with experimental data is satisfactory, the simulations are used to explain the experimental results and to study phenomena that could not be otherwise investigated with experimental techniques.

In the past few decades, MD has emerged as one of the most powerful tools to investigate the atomistic properties of lipid membranes because the structural characterization of lipid bilayers with atomic-level resolution is hindered by the high disorder of their physiological liquid-crystalline phase.

Several atomistic force-fields able to simulate lipid bilayers are currently available and under constant development; they have been able to satisfactorily reproduce several properties of lipid bilayers that can be measured experimentally using different diffraction or biophysical techniques, such as area per lipid, hydrophobic thickness, deuterium order parameters, or membrane elasticity (see [104] and http://nmrlipids.blogspot.fr).

Coarse-grain models, where several atoms are grouped together and represented by a single bead, and where the potential energy function is further simplified, are also under heavy development in the field of lipid membranes. They have been successfully used to interpret and predict large-scale membrane remodeling processes, including vesicle formation, membrane fusion, and membrane fission [105-107]. 


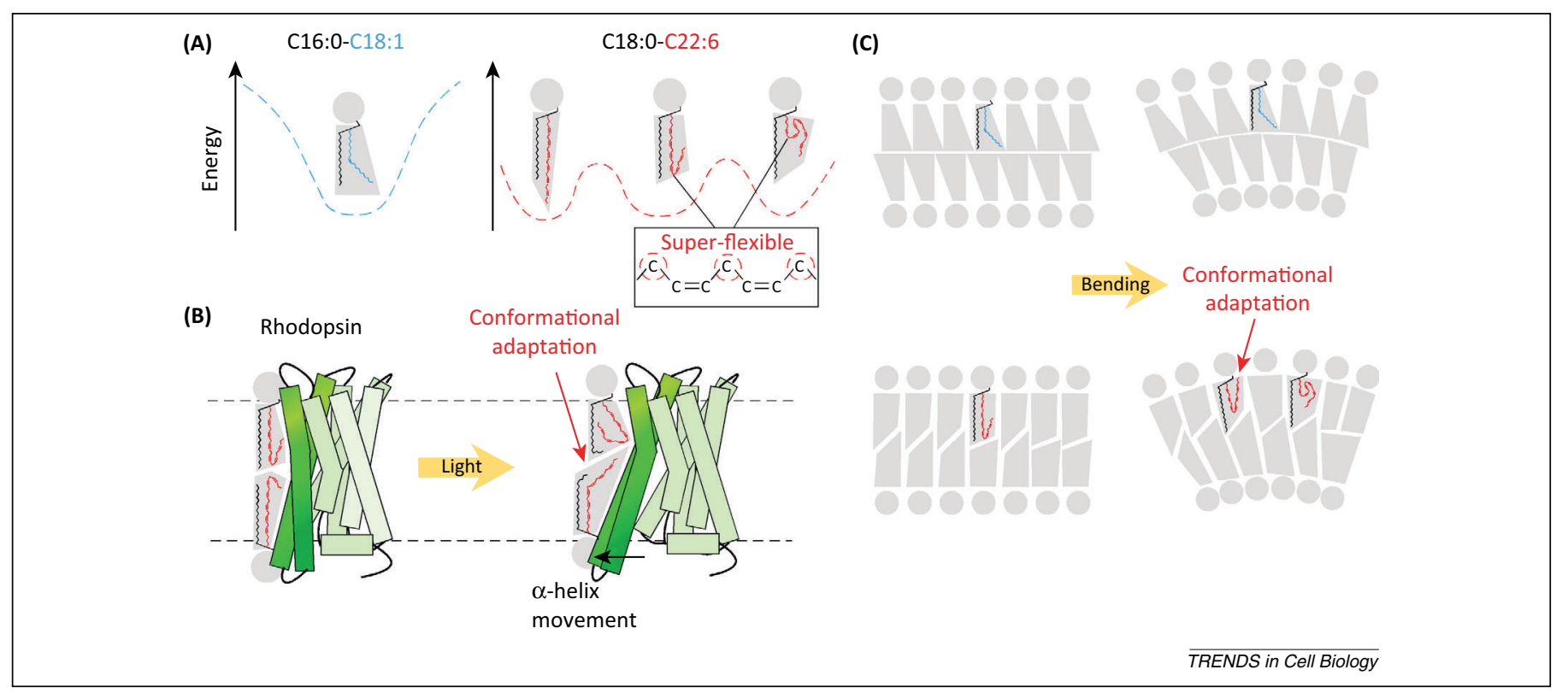

Figure 3. The conformational flexibility of polyunsaturated phospholipids (PLs) facilitates fast protein movements and membrane bending. (A) In contrast to monounsaturated acyl chains, which have a single optimal conformation, polyunsaturated acyl chains switch easily between different conformations of equivalent energy but drastically different geometry. (B) The flexibility of polyunsaturated PLs allows them to easily accommodate the sudden conformational change of proteins, such as the movements of the transmembrane helices from the GPCR rhodopsin depicted here. (C) Similarly, polyunsaturated PLs adapt their conformation to membrane shape, hence facilitating the mechanical action of proteins that bend and fission membranes.

because acyl chains undergo fast motions in bilayers (in the ns scale), biophysical approaches cannot give snapshots of their conformations but instead reveal trends as deduced from mean orientation and density measurements across the bilayer.

\section{Polyunsaturated PLs as contortionists}

Polyunsaturated acyl chains were initially considered to be more rigid than saturated acyl chains because a $\mathrm{C}=\mathrm{C}$ bond cannot rotate about its axis. In polyunsaturated acyl chains, however, the $\mathrm{C}=\mathrm{C}$ bonds are systematically flanked by two saturated bonds. Calculations and simulations revealed that this regular pattern of one non-rotating and two rotating bonds decreases the energy of rotation about the saturated carbons [32,67,68] (Figure 3A). The exceptional flexibility of polyunsaturated acyl chains gives a straightforward explanation for their benefit on the activation of rhodopsin and, more generally, on many membrane-embedded proteins as well as on the orientation of fluorescent probes [69-71] (Figure 3B). Polyunsaturated PLs quickly adapt their conformation to follow the rapid structural transitions of the rugged surface of transmembrane helix bundles [66,72].

\section{PL polyunsaturation in synaptic functions}

Synaptic vesicles (SV) deliver neurotransmitters in the synaptic cleft in response to action potentials. Although the abundance of polyunsaturated PLs is a remarkable feature of SVs [11] (Figure 1C), studies on the role of lipids in the cycling of these organelles have focused on other aspects [73]. In this section we present a hypothesis for the role of polyunsaturated PLs in SVs, which was inspired by observations made years ago but was formulated only recently [74].
In 1986, electron microscopy studies revealed that, among all exogenous factors that promote the formation of synaptic vesicles in cultures of fetal hypothalamic cells, the polyunsaturated fatty acids C20:4 and C22:6 were the most efficient [75]. The authors proposed that the features of SVs in PUFA-supplemented media, notably their regular shape and small diameter, as well as their abundance, reflect an enhancement of membrane fluidity in agreement with the model of Singer and Nicolson. Despite its prescience, this study was largely ignored. Later, other researchers reported that deficiency in polyunsaturated fatty acid synthesis in Caenorhabditis elegans leads to abnormally low levels of synaptic vesicles and defects in neurotransmitter release [76].

In 2000, physicists reported a striking effect of acyl chain polyunsaturation on the mechanical properties of giant liposomes made of synthetic PC. When PC contained at least one polyunsaturated acyl chain, the resistance of the bilayer against bending dropped abruptly by twofold, irrespective of the exact chemistry of the acyl chain [77].

Together, these seemingly disparate observations offer a straightforward explanation for the benefit of polyunsaturated PLs in SVs: these lipids would make the neuronal membrane more flexible and thus more prone to respond to the mechanical work exerted by proteins (Figure 3C). This would explain why SVs are the smallest membraneenclosed organelles (diameter $=40 \mathrm{~nm}$ ) and why their formation is so fast [78]. Recent experiments conducted with model lipid bilayers and with proteins involved in SV retrieval support this hypothesis [74]. First, the fission activity of the dynamin-endophilin complex, which forms a transient spiral at the neck of endocytic structures in the presence of GTP, increased dramatically upon the incorporation of C18:0-C22:6-n3 PLs at the expense of C16:0-C18:1 
PLs [74]. Second, the PM of non-neuronal cells that are forced to incorporate C22:6 in their PLs becomes more deformable by an external pulling force and more permissive to endophilin-driven endocytosis [74].

The shape and function of SVs and of photoreceptor discs are anything but similar. However, the benefits provided by polyunsaturated PLs in both membranes might originate from the same property: the ability of polyunsaturated chains to change their conformation (Figure 3B and 3C). Biochemical experiments and molecular dynamics simulations suggest that polyunsaturated PLs correct the lipid-packing defects induced by membrane curvature by filling the defects through their hyperflexible tail $[74,79,80]$. This finding gives a molecular explanation for the decrease in membrane bending rigidity [74,77], and may also explain some puzzling effects of polyunsaturated PLs on the targeting of lipidated proteins [81]. Moreover, it underlines a key qualitative difference between polyunsaturated PLs and other lipids. Whereas most lipids display an intrinsic shape (e.g., conical, cylindrical, or inversely conical), polyunsaturated PLs escape this classification. Like contortionists, they adopt different shapes of similar energy, thereby softening various membrane mechanical stresses (Figure 3A-C).

The proteins involved in SVs retrieval are well known, but the order in which they shape the synaptic membrane remains under intense investigation $[82,83]$. Recent studies suggest a two-step process in which endophilin and dynamin act first to promote the production of a transient endosome, which is then transformed into authentic SVs through the action of the AP2-clathrin coat [84-86]. The first step is super-fast [78] and, given the prominent role of endophilin and dynamin at this stage, it is tempting to associate this feature with the abundance of polyunsaturated PLs at the synapse [74].

Two recent studies nicely illustrate the effect of polyunsaturated PLs on membrane mechanics in other neuronal contexts. In $C$. elegans, mutating the enzymes involved in the synthesis of C20:4-n6 or in its incorporation into PLs causes a defect in touch sensation [87]. However, the defect is not complete, suggesting that polyunsaturated PLs merely make the membrane of sensory neurons more permissive to mechanotransduction. This effect could result from a reduction in the force threshold that leads to membrane deformation and/or from a facilitation of the conformational change of the channel. In Drosophila, manipulating the diet to decrease the amount of polyunsaturated PLs in the rhabdomere causes a decrease in the speed and gain of phototransduction [88]. Because this defect correlates with a stiffer membrane, this observation suggests that the membrane physical properties affect the gating of a mechanosensitive channel downstream of the phototransduction cascade. More generally, many channels exhibit strong sensitivity to both mechanical stress and lipid composition, and are excellent candidates to detect subtle changes in the hydrophobic membrane matrix [89-91].

\section{Concluding remarks}

Simple organisms contain only saturated and monounsaturated lipids, highlighting a fundamental role of the monounsaturated/saturated ratio for elementary functions. This ratio ranges from low values in membranes with a protective barrier function (apical membrane of epithelial cells, lung surfactant) [1], to high values in membranes with a biosynthetic function, as exemplified by the ER (Figure 1B). The evolutionary pressure for the conservation of $\Delta 9$ desaturase and the resulting selection of oleate (C18:1-n9) might then reflect an economical way to synthesize an acyl chain whose structure creates enough packing defects in membranes to host reactions associated with a huge transfer of material, such as the folding of transmembrane helices. Not surprisingly, this feature is hijacked by some pathogens, which take advantage of loose lipid-packing at the ER for their own replication [92].

The concepts that are operational to understand the differences between saturated and monounsaturated PLs (cylindrical vs conical shape, tight vs loose packing, barrier vs biogenic function) are not adapted to understanding the properties of polyunsaturated PLs. Functionally, the hallmark of these PLs is their abundance in membranes that host super-fast and efficient reactions (e.g., phototransduction, ATP synthesis, neurotransmission). Molecularly, their distinguishable feature is their fast adaptation: like contortionists, they are alternatively cones, cylinders, or hairpins to minimize the packing stress that results from the fast switch of transmembrane proteins or the mechanical work of membrane-bending proteins. In other words, polyunsaturated PLs are not a type of 'super' monounsaturated PLs; they are just different.

The examples evoked here might help in understanding the traits imparted by saturated, monounsaturated and polyunsaturated lipids in other organisms, cells, or organelles (Box 3). Such studies will require careful lipid analysis, notably at the subcellular level, as well as sensitive functional assays and tedious reconstitution experiments, notably of structural fission and fusion intermediates [93], because the effects are likely to be subtler than those observed by the deletion of essential proteins. The nematode C. elegans, which is amenable to genetic manipulation and displays a large set of enzymes for lipid remodeling, provides a useful model [7,76,78,94-96]. In addition, recent advances in gene-editing technology open the possibility of comprehensive studies of the impact of lipid remodeling in cultured cells. Another challenge is to determine the spatial and temporal control of lipid remodeling at the subcellular level: most lipid remodeling enzymes have been reported to

\section{Box 3. Outstanding issues}

- Lysophospholipid acyltransferase specificity, regulation and structure

- Spatiotemporal control of PL acyl chain remodeling

- Control of the monounsaturated/saturated PL ratio along the secretory pathway

- Origin of the gradient of polyunsaturated PLs along axons

- Structure of polyunsaturated PLs in fission and fusion intermediates

- Correlation between fast endocytosis at the synapse and polyunsaturated PLs

- Differences between $\omega 3$ and $\omega 6$ PLs in synaptic functions

- Impact of the acyl chain remodeling of phosphoinositides on cancer cells 
localize at the ER, but their lipid products clearly concentrate at specific locations. Last, we are far from understanding all subtle changes that distinguish fatty acyl chains in PLs. For example, the difference between $\omega 3$ and $\omega 6$ PLs is not clear-cut: both seem prone to rapid conformational changes, but the current experimental data and simulations are fragmentary $[65,66,97,98]$.

Counterexamples of the general trends of acyl chain distribution will also deserve special attention. The ER is not made exclusively of monounsaturated lipids: long saturated lipids might participate in the creation of diffusion barriers important for the segregation of proteins [99]. Conversely, monounsaturated lipids in the exoplasmic leaflet of the PM membrane are privileged targets for toxins that drive membrane invagination [100]. Overall, lipid species whose acyl chains differ in length and unsaturation from bulk lipids are more likely to have specific roles, a prominent example being phosphoinositides (PI) [94,101]. During nuclear envelope formation, polyunsaturated PIs are crucial for membrane fusion events [102]. In cancer cell lines, a marked shift in the length and unsaturation of PIs has been reported recently, but its impact on cellular functions is unknown [24].

\section{Acknowledgments}

Work in the laboratory of B.A. is supported by the CNRS, the European Research Council (advanced grant 268888), and the Agence Nationale de la Recherche (ANR-11-LABX-0028-01).

\section{References}

1 Sampaio, J.L. et al. (2011) Membrane lipidome of an epithelial cell line. Proc. Natl. Acad. Sci. U.S.A. 108, 1903-1907

2 Bigay, J. and Antonny, B. (2012) Curvature, lipid packing, and electrostatics of membrane organelles: defining cellular territories in determining specificity. Dev. Cell 23, 886-895

3 Brugger, B. (2014) Lipidomics: analysis of the lipid composition of cells and subcellular organelles by electrospray ionization mass spectrometry. Annu. Rev. Biochem. 83, 79-98

4 Holthuis, J.C. and Menon, A.K. (2014) Lipid landscapes and pipelines in membrane homeostasis. Nature 510, 48-57

5 Harayama, T.et al. (2014) Lysophospholipid acyltransferases mediate phosphatidylcholine diversification to achieve the physical properties required in vivo. Cell Metab. 20, 295-305

6 Lemmon, M.A. (2008) Membrane recognition by phospholipid-binding domains. Nat. Rev. Mol. Cell Biol. 9, 99-111

7 Wallis, J.G. et al. (2002) Polyunsaturated fatty acid synthesis: what will they think of next? Trends Biochem. Sci. 27, 467

8 Keenan, T.W. and Morre, D.J. (1970) Phospholipid class and fatty acid composition of golgi apparatus isolated from rat liver and comparison with other cell fractions. Biochemistry 9, 19-25

9 Schneiter, R. et al. (1999) Electrospray ionization tandem mass spectrometry (ESI-MS/MS) analysis of the lipid molecular species composition of yeast subcellular membranes reveals acyl chainbased sorting/remodeling of distinct molecular species en route to the plasma membrane. J. Cell Biol. 146, 741-754

10 Salem, N., Jr et al. (2001) Mechanisms of action of docosahexaenoic acid in the nervous system. Lipids 36, 945-959

11 Takamori, S. et al. (2006) Molecular anatomy of a trafficking organelle. Cell 127, 831-846

12 Yang, H.J. et al. (2012) Axonal gradient of arachidonic acid-containing phosphatidylcholine and its dependence on actin dynamics. J. Biol. Chem. 287, 5290-5300

13 Smith, W. and Mukhopadhyay, R. (2012) Essential fatty acids: the work of George and Mildred Burr. J. Biol. Chem. 287, 35439-35441

14 Bazinet, R.P. and Laye, S. (2014) Polyunsaturated fatty acids and their metabolites in brain function and disease. Nat. Rev. Neurosci. $15,771-785$
15 Shindou, H. et al. (2013) Generation of membrane diversity by lysophospholipid acyltransferases. J. Biochem. 154, 21-28

16 Watts, J.L. and Browse, J. (2002) Genetic dissection of polyunsaturated fatty acid synthesis in Caenorhabditis elegans. Proc. Natl. Acad. Sci. U.S.A. 99, 5854-5859

17 Nguyen, L.N. et al. (2014) Mfsd2a is a transporter for the essential omega-3 fatty acid docosahexaenoic acid. Nature 509, 503-506

18 Shindou, H. and Shimizu, T. (2009) Acyl-CoA:lysophospholipid acyltransferases. J. Bio. Chem. 284, 1-5

19 Schlame, M. et al. (2012) The physical state of lipid substrates provides transacylation specificity for tafazzin. Nat. Chem. Biol. 8, 862-869

20 Connor, W.E. et al. (1998) Uneven distribution of desmosterol and docosahexaenoic acid in the heads and tails of monkey sperm. J. Lipid Res. 39, 1404-1411

21 Koeberle, A. et al. (2013) Arachidonoyl-phosphatidylcholine oscillates during the cell cycle and counteracts proliferation by suppressing Akt membrane binding. Proc. Natl. Acad. Sci. U.S.A. $110,2546-2551$

22 Atilla-Gokcumen, G.E. et al. (2014) Dividing cells regulate their lipid composition and localization. Cell 156, 428-439

23 Guo, S. et al. (2014) Significantly increased monounsaturated lipids relative to polyunsaturated lipids in six types of cancer microenvironment are observed by mass spectrometry imaging. Sci. Rep. 4, 5959

24 Naguib, A. et al. (2015) P53 mutations change phosphatidylinositol acyl chain composition. Cell Rep. 10, 8-19

25 Kincaid, M.M. and Cooper, A.A. (2007) ERADicate ER stress or die trying. Antioxid. Redox Signal. 9, 2373-2387

26 Karaskov, E. et al. (2006) Chronic palmitate but not oleate exposure induces endoplasmic reticulum stress, which may contribute to INS-1 pancreatic beta-cell apoptosis. Endocrinology 147, 3398-3407

27 Bertolotti, A. et al. (2000) Dynamic interaction of BiP and ER stress transducers in the unfolded-protein response. Nat. Cell Biol. 2, 326-332

28 Gardner, B.M. and Walter, P. (2011) Unfolded proteins are Ire1activating ligands that directly induce the unfolded protein response. Science 333, 1891-1894

29 Diakogiannaki, E. et al. (2008) Differential regulation of the endoplasmic reticulum stress response in pancreatic beta-cells exposed to long-chain saturated and monounsaturated fatty acids. J. Endocrinol. 197, 553-563

30 Cunha, D.A. et al. (2008) Initiation and execution of lipotoxic ER stress in pancreatic $\beta$-cells. J. Cell Sci. 121, 2308-2318

31 Ariyama, H. et al. (2010) Decrease in membrane phospholipid unsaturation induces unfolded protein response. J. Biol. Chem. 285, 22027-22035

32 Deguil, J. et al. (2011) Modulation of lipid-induced ER stress by fatty acid shape. Traffic 12, 349-362

33 Dhayal, S. and Morgan, N.G. (2011) Structure-activity relationships influencing lipid-induced changes in eIF2alpha phosphorylation and cell viability in BRIN-BD11 cells. FEBS Lett. 585, 2243-2248

34 Achard, C.S. and Laybutt, D.R. (2012) Lipid-induced endoplasmic reticulum stress in liver cells results in two distinct outcomes: adaptation with enhanced insulin signaling or insulin resistance. Endocrinology 153, 2164-2177

35 Busch, A.K. et al. (2005) Increased fatty acid desaturation and enhanced expression of stearoyl coenzyme a desaturase protects pancreatic $\beta$-cells from lipoapoptosis. Diabetes 54, 2917-2924

36 Borradaile, N.M. et al. (2006) Disruption of endoplasmic reticulum structure and integrity in lipotoxic cell death. J. Lipid Res. 47, 27262737

37 Pineau, L. et al. (2009) Lipid-induced ER stress: synergistic effects of sterols and saturated fatty acids. Traffic 10, 673-690

38 Gwiazda, K.S. et al. (2009) Effects of palmitate on ER and cytosolic $\mathrm{Ca}^{2+}$ homeostasis in $\beta$-cells. Am. J. Physiol. Endocrinol. Metab. 296, E690-E701

$39 \mathrm{Li}, \mathrm{Y}$. et al. (2004) Enrichment of endoplasmic reticulum with cholesterol Inhibits sarcoplasmic-endoplasmic reticulum calcium ATPase-2b activity in parallel with increased order of membrane lipids: implications for depletion of endoplasmic reticulum calcium stores and apoptosis in cholesterol-loaded macrophages. J. Biol. Chem. 279, 37030-37039 
40 Akerfeldt, M.C. et al. (2008) Cytokine-induced beta-cell death is independent of endoplasmic reticulum stress signaling. Diabetes 57, 3034-3044

41 Choi, S-E. et al. (2008) A chemical chaperone 4-PBA ameliorates palmitate-induced inhibition of glucose-stimulated insulin secretion (GSIS). Arch. Biochem. Biophys. 475, 109-114

42 Ozcan, U. et al. (2006) Chemical chaperones reduce ER stress and restore glucose homeostasis in a mouse model of type 2 diabetes. Science 313, 1137-1140

43 Nilsson, I. et al. (2001) Inhibition of protein translocation across the endoplasmic reticulum membrane by sterols. J. Biol. Chem. 276, 41748-41754

44 Michalak, M. et al. (2002) $\mathrm{Ca}^{2+}$ signaling and calcium binding chaperones of the endoplasmic reticulum. Cell Calcium 32, 269-278

45 Volmer, R. et al. (2013) Membrane lipid saturation activates endoplasmic reticulum unfolded protein response transducers through their transmembrane domains. Proc. Natl. Acad. Sci. U.S.A. 110, 4628-4633

46 Hou, N.S. et al. (2014) Activation of the endoplasmic reticulum unfolded protein response by lipid disequilibrium without disturbed proteostasis in vivo. Proc. Natl. Acad. Sci. U.S.A. 111 E2271-E2280

47 Kitai, Y. et al. (2013) Membrane lipid saturation activates IRE1alpha without inducing clustering. Genes Cells 18, 798-809

48 Surma, M.A. et al. (2013) A lipid E-MAP identifies Ubx2 as a critical regulator of lipid saturation and lipid bilayer stress. Mol. Cell 51, 519 530

49 Volmer, R. and Ron, D. (2014) Lipid-dependent regulation of the unfolded protein response. Curr. Opin. Cell Biol. 33C, 67-73

50 Antonny, B. (2011) Mechanisms of membrane curvature sensing. Annu. Rev. Biochem. 80, 101-123

51 Nath, S. et al. (2014) Lipidation of the LC3/GABARAP family of autophagy proteins relies on a membrane-curvature-sensing domain in Atg3. Nat. Cell Biol. 16, 415-424

52 Drin, G. et al. (2007) A general amphipathic alpha-helical motif for sensing membrane curvature. Nat. Struct. Mol. Biol. 14, 138-146

53 Hatzakis, N.S. et al. (2009) How curved membranes recruit amphipathic helices and protein anchoring motifs. Nat. Chem. Biol. 5, 835-841

54 Vanni, S. et al. (2014) A sub-nanometre view of how membrane curvature and composition modulate lipid packing and protein recruitment. Nat. Commun. 5, 4916

55 Ogasawara, Y. et al. (2014) Stearoyl-CoA desaturase 1 activity is required for autophagosome formation. J. Biol. Chem. 289, 2393823950

56 Preston, A.M. et al. (2009) Reduced endoplasmic reticulum (ER)-toGolgi protein trafficking contributes to ER stress in lipotoxic mouse beta cells by promoting protein overload. Diabetologia 52, 2369-2373

57 Payet, L.A. et al. (2013) Saturated fatty acids alter the late secretory pathway by modulating membrane properties. Traffic 14, 1228-1241

58 Cui, H. et al. (2011) Mechanism of membrane curvature sensing by amphipathic helix containing proteins. Biophys. J. 100, 1271-1279

59 Vamparys, L. et al. (2013) Conical lipids in flat bilayers induce packing defects similar to that induced by positive curvature. Biophys. J. 104, 585-593

60 Vanni, S. et al. (2013) Amphipathic lipid packing sensor motifs: probing bilayer defects with hydrophobic residues. Biophys. J. 104, 575-584

61 Campelo, F. and Kozlov, M.M. (2014) Sensing membrane stresses by protein insertions. PLoS Comput. Biol. 10, e1003556

62 Stone, W.L. et al. (1979) A reinvestigation of the fatty acid content of bovine, rat and frog retinal rod outer segments. Exp. Eye Res. 28, 387-397

63 Mitchell, D.C. et al. (2001) Optimization of receptor-G protein coupling by bilayer lipid composition I: kinetics of rhodopsintransducin binding. J. Biol. Chem. 276, 42801-42806

64 Niu, S.L. et al. (2004) Reduced G protein-coupled signaling efficiency in retinal rod outer segments in response to n-3 fatty acid deficiency. J. Biol. Chem. 279, 31098-31104

65 Rajamoorthi, K. et al. (2005) Packing and viscoelasticity of polyunsaturated omega- 3 and omega- 6 lipid bilayers as seen by ${ }^{2} \mathrm{H}$ NMR and X-ray diffraction. J. Am. Chem. Soc. 127, 1576-1588
66 Eldho, N.V. et al. (2003) Polyunsaturated docosahexaenoic vs docosapentaenoic acid-differences in lipid matrix properties from the loss of one double bond. J. Am. Chem. Soc. 125, 64096421

67 Applegate, K.R. and Glomset, J.A. (1986) Computer-based modeling of the conformation and packing properties of docosahexaenoic acid. $J$. Lipid Res. 27, 658-680

68 Feller, S.E. et al. (2002) Polyunsaturated fatty acids in lipid bilayers: intrinsic and environmental contributions to their unique physical properties. J. Am. Chem. Soc. 124, 318-326

69 Mitchell, D.C. and Litman, B.J. (1998) Molecular order and dynamics in bilayers consisting of highly polyunsaturated phospholipids. Biophys. J. 74, 879-891

70 Grossfield, A. et al. (2006) A role for direct interactions in the modulation of rhodopsin by omega-3 polyunsaturated lipids. Proc. Natl. Acad. Sci U.S.A. 103, 4888-4893

71 Soubias, O. et al. (2006) Evidence for specificity in lipid-rhodopsin interactions. J. Biol. Chem. 281, 33233-33241

72 Soubias, O. et al. (2010) Contribution of membrane elastic energy to rhodopsin function. Biophys. J. 99, 817-824

73 Puchkov, D. and Haucke, V. (2013) Greasing the synaptic vesicle cycle by membrane lipids. Trends Cell Biol. 23, 493-503

74 Pinot, M. et al. (2014) Polyunsaturated phospholipids facilitate membrane deformation and fission by endocytic proteins. Science 345, 693-697

75 Tixier-Vidal, A. et al. (1986) Effects of polyunsaturated fatty acids and hormones on synaptogenesis in serum-free medium cultures of mouse fetal hypothalamic cells. Neuroscience 17, 115-132

76 Lesa, G.M. et al. (2003) Long chain polyunsaturated fatty acids are required for efficient neurotransmission in C. elegans. J. Cell Sci. 116 , 4965-4975

77 Rawicz, W. et al. (2000) Effect of chain length and unsaturation on elasticity of lipid bilayers. Biophys. J. 79, 328-339

78 Watanabe, S. et al. (2013) Ultrafast endocytosis at Caenorhabditis elegans neuromuscular junctions. Elife 2, e00723

79 Risselada, H.J. and Marrink, S.J. (2009) Curvature effects on lipid packing and dynamics in liposomes revealed by coarse grained molecular dynamics simulations. Phys. Chem. Chem. Phys. 11, 2056-2067

80 Hishikawa, D. et al. (2013) Identification of Sec14-like 3 as a novel lipid-packing sensor in the lung. FASEB J. 27, 5131-5140

81 Seo, J. et al. (2006) Docosahexaenoic acid selectively inhibits plasma membrane targeting of lipidated proteins. FASEB J. 20, 770

82 Saheki, Y. and De Camilli, P. (2012) Synaptic vesicle endocytosis. Cold Spring Harb. Perspect. Biol. 4, a005645

83 Kononenko, N.L. and Haucke, V. (2015) Molecular mechanisms of presynaptic membrane retrieval and synaptic vesicle reformation. Neuron 85, 484-496

84 Boucrot, E. et al. (2015) Endophilin marks and controls a clathrinindependent endocytic pathway. Nature 517, 460-465

85 Watanabe, S. et al. (2014) Clathrin regenerates synaptic vesicles from endosomes. Nature 515, 228-233

86 Kononenko, N.L. et al. (2014) Clathrin/AP-2 mediate synaptic vesicle reformation from endosome-like vacuoles but are not essential for membrane retrieval at central synapses. Neuron 82, 981-988

87 Vasquez, V. et al. (2014) Phospholipids that contain polyunsaturated fatty acids enhance neuronal cell mechanics and touch sensation. Cell Rep. 6, 70-80

88 Randall, A.S. et al. (2015) Speed and sensitivity of phototransduction in Drosophila depend on degree of saturation of membrane phospholipids. J. Neurosci. 35, 2731-2746

89 Nilius, B. and Honore, E. (2012) Sensing pressure with ion channels. Trends Neurosci. 35, 477-486

90 Fink, M. et al. (1998) A neuronal two P domain $\mathrm{K}^{+}$channel stimulated by arachidonic acid and polyunsaturated fatty acids. EMBO J. 17, 3297-3308

91 Brohawn, S.G. et al. (2014) Physical mechanism for gating and mechanosensitivity of the human TRAAK $\mathrm{K}^{+}$channel. Nature 516, $126-130$ 
92 Lyn, R.K. et al. (2014) Stearoyl-CoA desaturase inhibition blocks formation of hepatitis C virus-induced specialized membranes. Sci. Rep. 4, 4549

93 Aeffner, S. et al. (2012) Energetics of stalk intermediates in membrane fusion are controlled by lipid composition. Proc. Natl. Acad. Sci U.S.A 109, E1609-E1618

94 Lee, H.C. et al. (2008) Caenorhabditis elegans mboa-7, a member of the MBOAT family, is required for selective incorporation of polyunsaturated fatty acids into phosphatidylinositol. Mol. Biol. Cell 19, 1174-1184

95 Marza, E. and Lesa, G.M. (2006) Polyunsaturated fatty acids and neurotransmission in Caenorhabditis elegans. Biochem. Soc. Trans. 34, 77-80

96 Entchev, E.V. et al. (2008) LET-767 is required for the production of branched chain and long chain fatty acids in Caenorhabditis elegans. J. Biol. Chem. 283, 17550-17560

97 Deng, Y. et al. (2009) Docosapentaenoic acid (DPA) is a critical determinant of cubic membrane formation in amoeba Chaos mitochondria. FASEB J. 23, 2866-2871

98 Mitchell, D.C. et al. (2012) Quantifying the differential effects of DHA and DPA on the early events in visual signal transduction. Chem. Phys. Lipids 165, 393-400

99 Clay, L. et al. (2014) A sphingolipid-dependent diffusion barrier confines ER stress to the yeast mother cell. Elife 3, e01883
100 Romer, W. et al. (2007) Shiga toxin induces tubular membrane invaginations for its uptake into cells. Nature 450, 670-675

101 D'Souza, K. and Epand, R.M. (2014) Enrichment of phosphatidylinositols with specific acyl chains. Biochim. Biophys. Acta 1838, 1501-1508

102 Zhendre, V. et al. (2011) Key role of polyphosphoinositides in dynamics of fusogenic nuclear membrane vesicles. PLoS ONE 6, e23859

103 Morimoto, R. et al. (2014) Rapid production of platelet-activating factor is induced by protein kinase Calpha-mediated phosphorylation of lysophosphatidylcholine acyltransferase 2 protein. J. Biol. Chem. 289, 15566-15576

104 Piggot, T.J. et al. (2012) Molecular dynamics simulations of phosphatidylcholine membranes: a comparative force field study. $J$. Chem. Theor. Comput. 8, 4593-4609

105 Marrink, S.J. and Tieleman, D.P. (2013) Perspective on the Martini model. Chem. Soc. Rev. 42, 6801-6822

106 Wang, Z.J. and Deserno, M. (2010) A systematically coarse-grained solvent-free model for quantitative phospholipid bilayer simulations. J. Phys. Chem. B 114, 11207-11220

107 Shinoda, W. et al. (2010) Zwitterionic lipid assemblies: molecular dynamics studies of monolayers, bilayers, and vesicles using a new coarse grain force field. J. Phys. Chem. B 114, 68366849 